\title{
ON CONDITIONING FOR A GENERAL SYSTEM OF FOUR POINT BOUNDARY VALUE PROBLEMS
}

\author{
M. S. N. MURTY
}

\begin{abstract}
In this paper we investigate the close relationships between the stability constants and the growth behaviour of the fundamental matrix to the general FPBVP'S associated with the general first order matrix differential equation.
\end{abstract}

\section{Introduction}

Boundary value problems play an important role in a variety of real world problems. In finding solutions to boundary value problems, the construction of Green's matrix is vital. It is well known that the construction of Green's matrix plays an important role in solving problems involving non-singular matrices $([1],[4]))$. In this paper we consider the four point boundary value problem (FPBVP) associated with the general first order matrix differential equations of the form

$$
L y \equiv P(t) y^{\prime}+Q(t) y=f(t), \quad a \leq t \leq d
$$

where $P, Q \in\left[L_{p}(a, d)\right]^{n \times n}, \quad f \in\left[L_{p}(a, d)\right]^{n}$ for some $p$ satisfying $(1 \leq p<\infty)$ satisfying the boundary condition

$$
B y \equiv M y(a)+N y(b)+R y(c)+S y(d)=\alpha
$$

where $M, N, R, S \in R^{n \times n}$ and $\alpha \in R^{n}$, and $y(t)$ is a column matrix with components $\left(y_{1}, y_{2}, \ldots, y_{n}\right)$ and we assume throughout this paper that $P(t)$ is a non-singular square matrix on $[a, d]$.

The condition numbers in principle indicate by how much any possible error in the boundary conditions may be amplified; it turns out that they also play an important

Received Janu 16, 1994

1991 Mathematics Subject Classification. 34B27

Key words and phrases. Boundary value problems, slability, conditioning, Green's matrix. 
role in estimating the global error due to small perturbations. In this direction Matheja [2], Murty and Sivasundaram [3] have obtained results for two and three point boundary value problems associated with linear and non-linear differential equations. In this paper we investigate the close relationships between the stability constants and the growth behavior of the fundamental matrix to the general FPBVPs. Moreover we show that the conditioning number is the right criterion to indicate possible error amplifications of the perturbed boundary conditions.

\section{Main results}

The problem (1.1) and (1.2) is called invertible if the homogeneous BVP corresponding to (1.1) and (1.2) with $f=0$ and $\alpha=0$ has only the trivial solution $y=0$. Evidently the FPBVP (1.1) and (1.2) has a unique solution $y$ if and only if the characteristic matrix $D=M Y(a)+N Y(b)+R Y(c)+S Y(d)$ is non-singular where $Y$ is a fundamental matrix for $L y=0$. In this case the general solution is given by

$$
y(t)=Y(t) D^{-1} \alpha+\int_{a}^{d} G(t, s) f(s) d s
$$

where $G$ is the Green's matrix for the homogeneous BVP given by

$$
\begin{aligned}
& G(t, s)= \\
& t \in[a, b] \\
& \left\{\begin{array}{l}
Y(t) D^{-1} M Y(a) Y^{-1}(s) P^{-1}(s) \quad a \leq s \leq t \leq b<c<d \\
-Y(t) D^{-1}[N Y(b)+R Y(c)+S Y(d)] Y^{-1}(s) P^{-1}(s) \quad a \leq t<s \leq b<c<d \\
-Y(t) D^{-1}[R Y(c)+S Y(d)] Y^{-1}(s) P^{-1}(s) \quad a<t<b<s<c<d \\
-Y(t) D^{-1} S Y(d) Y^{-1}(s) P^{-1}(s) \quad a<t<b<c<s<d
\end{array}\right.
\end{aligned}
$$

and Green's matrices for the other two intervals $t \in[b, c]$ and $t \in[c, d]$ may be written in a similar way. Thus in principle, a knowledge of any fundamental matrix for $L y=0$ enables us to calculate the Green's matrix and inturn the solution $y$ of the FPBVP given by $(2.1)$.

We shall now see how expression (2.1) can be used to examine the conditioning of (1.1) and (1.2). Since we shall be using orthogonal transformations we take (1.1) to be the Euclidian norm in $R^{n}$. It is a well known fact that all norms on $R^{n}$ are almost equivalent and the use of another norm changes only the numerical constants in the subsequent results and hence we make use of the following notation.

Let

$$
\|u\|_{p}=\left[\int_{a}^{d}|u(s)|^{p} d s\right]^{1 / p}, \quad 1 \leq p<\infty
$$

and

$$
\|u\|=S u p_{t \in[a, d]}|u(t)|
$$


be its limiting value as $p \rightarrow \infty$. Then we have from (2.1)

$$
\begin{gathered}
\|y\|=\|y\|_{\infty} \leq \beta|B y|+\gamma_{q}\|L y\|_{p}, \quad \frac{1}{p}+\frac{1}{q}=1 \\
\beta=\left\|Y(t) D^{-1}\right\| \\
\gamma_{q}=\operatorname{Sup}_{t}\left[\int_{a}^{d}[G(t, s)]^{q} d s\right]^{1 / q}
\end{gathered}
$$

The selection of suitable norm in (2.4) depends upon the problem under consideration. For the sake of convenience we consider the case of $p=1$. When $p=1,(2.3),(2.4)$, and (2.5) reduces to

$$
\begin{gathered}
\|y\|_{\infty} \leq \beta|\alpha|+\gamma\|f\| \\
\beta=\left\|y(t) D^{-1}\right\|
\end{gathered}
$$

and

$$
\gamma=S u p_{t, s}|G(t, s)|
$$

If in addition, we assume that the boundary conditions are scaled in such a way that

$$
M M^{T}+N N^{T}+R R^{T}+S S^{T}=I,
$$

then

$$
\begin{aligned}
\left|Y(t) D^{-1}\right|= & \mid G(t, a) G^{T}(t, a) \\
& +G(t, b) G^{T}(t, b)+G(t, c) G^{T}(t, c)+G(t, d) G^{T}(t, d) \mid
\end{aligned}
$$

and hence

$$
\beta^{2} \leq \gamma^{2}+\gamma^{2}+\gamma^{2}+\gamma^{2} \leq 4 \gamma^{2}, \quad \beta \leq 2 \gamma .
$$

Thus in this case the stability constant $\gamma$ gives a measure for the sensitivity of (1.1) and (1.2) to changes in the data. We note from (2.7) and (2.8) that both the fundamental solution and the boundary conditions (1.2) will actually determine the magnitude of the stability constants $\beta$ and $\gamma$. Thus it is possible to construct systems for which no boundary conditions exist such that $\beta$ and $\gamma$ are of moderate size: it is also possible to find boundary conditions for (1.1) so that $\beta$ and $\gamma$ are large. Thus if the system (1.1) can support a well conditioned problem then the conditioning is intimately related to the choice of the boundary conditions. It is only for this reason that initial value methods such as shooting methods may fail to given fair results for some well conditioned problems.

Definition 2.1. The condition number $\beta$ of the boundary value problem (1.1) and (1.2) is defined as

$$
\beta=S u p_{a \leq t \leq d}\left\|Y(t) D^{-1}\right\| .
$$

The number $\beta$ is independent of the choice of the fundamental matrix. It is easily seen that if $Z(t)$ is another fundamental matrix of $L y=0$ then there exists a constant nonsingular matrix $C$ such that $Z(t)=Y(t) C$. 
We consider the variation $y(t)$ of (1.1) with respect to the small perturbations in the boundary conditions. For this we consider the perturbation of $(1.2)$ in the form

$$
(M+\delta M) y(a)+(N+\delta N) y(b)+(R+\delta R) y(c)+(S+\delta S) y(d)=\alpha+\delta \alpha
$$

We also assume that the perturbations are such that the characteristic matrix

$$
D_{1}=D+\delta D=(M+\delta M) Y(a)+(N+\delta N) Y(b)+(R+\delta R) Y(c)+(S+\delta S) Y(d)
$$

is non-singular.

The following lemma shows that $\left\|\delta D D^{-1}\right\|$ can be estimated in terms of $\beta$ and the perturbations.

\section{Lemma 2.1.}

$$
\left\|\delta D D^{-1}\right\| \leq(\|\delta M\|+\|\delta N\|+\|\delta R\|+\|\delta s\|) \beta .
$$

\section{Proof.}

$$
\begin{aligned}
\left\|\delta D D^{-1}\right\| & \leq\|\delta M\|\left\|Y(a) D^{-1}\right\|+\|\delta N\|\left\|Y(b) D^{-1}\right\|+\|\delta R\|\left\|Y(c) D^{-1}\right\|+\|\delta s\| Y(d) D^{-1} \| \\
& \leq(\|\delta M\|+\|\delta N\|+\|\delta R\|+\|\delta s\|)\left\|Y(t) D^{-1}\right\|, \\
& \leq(\|\delta M\|+\|\delta N\|+\|\delta R\|+\|\delta s\|) \beta
\end{aligned}
$$

Theorem 2.1. Let $\epsilon>0$ be such that $0<\epsilon<\left[\frac{1}{(1+k) \delta \beta}\right]$, where

$$
\delta=\max \{\|\delta M\|,\|\delta N\|, 2\|\delta R\|, 2\|\delta s\|,\|\delta \alpha\|,\|\delta D\|\}
$$

and

$$
K=\max \int_{a}^{d}\left\|Y^{-1}(s) P^{-1}(s) f(s)\right\| d s .
$$

Then the solution $y^{*}(t)$ of (1.1) satisfying (2.9) is such that

$$
\begin{aligned}
& \delta \beta[1-K(\|Y(a)\|+\|Y(b)\|+2\|Y(c)\|+3\|Y(d)\|)] \\
\leq & \max _{t \in[a, d]}\|y *(t)-y(t)\| \\
\leq & \delta \beta[1+K(\|y(a)\|+\|Y(b)\|+2\|Y(c)\|+3\|Y(d)\|)] .
\end{aligned}
$$

Proof. We shall discuss the case only when $s \in[a, b]$, the other cases can be dealt with similarly. Any solution $y *(t)$ of (1.1) satisfying (2.9) is given by

$$
y *(t)=Y(t) D_{1}^{-1}(\alpha+\delta \alpha)+\int_{a}^{d} G_{1}(t, s) f(s) d s
$$


where

$$
\begin{aligned}
& G_{1}(t, s)= \\
& \left\{\begin{array}{l}
Y(t) D_{1}^{-1} M_{1} Y(a) Y^{-1}(s) P^{-1}(s), \quad a \leq s<t<b<c<d \\
-Y(t) D_{1}^{-1}\left[N_{1} Y(b)+R_{1} Y(c)+S_{1} Y(d)\right] Y^{-1}(s) P^{-1}(s), \quad a \leq t<s<b<c<d \\
-Y(t) D_{1}^{-1}\left[R_{1} Y(c)+S_{1} Y(d)\right] Y^{-1}(s) P^{-1}(s), \quad a<t<b<s<c<d \\
-Y(t) D_{1}^{-1} S_{1} Y(d) Y^{-1}(s) P^{-1}(s), \quad a<t<b<c<s<d,
\end{array}\right.
\end{aligned}
$$

and Green's matrices for the other two cases namely $t \in[b, c]$ and $t \in[c, d]$ can be written in a similar manner, where

$$
M_{1}=M+\delta M, \quad N_{1}=N+\delta N, \quad R_{1}=R+\delta R \quad \text { and } \quad S_{1}=S+\delta S
$$

\section{Consider}

$$
\begin{aligned}
\left\|y^{*}(t)-y(t)\right\| \leq & \left\|Y(t)\left[D_{1}^{-1}(\alpha+\delta \alpha)-D^{-1} \alpha\right]\right\| \\
& +\int_{a}^{t}\left\|Y(t)\left[D_{1}^{-1} M_{1}-D^{-1} M\right] Y(a) Y^{-1}(s) P^{-1}(s) f(s)\right\| d s \\
& +\int_{t}^{b} \| Y(t)\left[D_{1}^{-1}\left(N_{1} Y(b)+R_{1} Y(c)+S_{1} Y(d)\right)\right. \\
& \left.-D^{-1}(N Y(b)+R Y(c)+S Y(d))\right] Y^{-1}(s) P^{-1}(s) f(s) \| d s \\
& +\int_{b}^{c} \| Y(t)\left[D_{1}^{-1}\left(R_{1} Y(c)+S_{1} Y(d)\right)\right. \\
& \left.-D^{-1}(R Y(c)+S Y(d))\right] Y^{-1}(s) P^{-1}(s) f(s) \| d s \\
& +\int_{c}^{d}\left\|Y(t)\left[D_{1}^{-1} S_{1}-D^{-1} S\right] Y(d) Y^{-1}(s) P^{-1}(s) f(s)\right\| d s .
\end{aligned}
$$

We make use of the following rough estimates:

$$
\begin{aligned}
D_{1}^{-1}(\alpha+\delta \alpha)-D^{-1} \alpha & =(D+\delta D)^{-1}(\alpha+\delta \alpha)-D^{-1} \alpha \\
& =D^{-1}\left(I+D^{-1} \delta D\right)^{-1}(\alpha+\delta \alpha)-D^{-1} \alpha \\
& \simeq D^{-1}\left[I-D^{-1} \delta D\right](\alpha+\delta \alpha)-D^{-1} \alpha \simeq D^{-1} \alpha
\end{aligned}
$$

Similarly

$$
\begin{aligned}
& D_{1}^{-1} M_{1}-D^{-1} M \simeq D^{-1} \delta M, D_{1}^{-1} N_{1}-D^{-1} N \simeq D^{-1} \delta N \\
& D_{1}^{-1} R_{1}-D^{-1} R \simeq D^{-1} \simeq \delta R \text { and } D_{1}^{-1} S_{1}-D^{-1} S \simeq D^{-1} \delta S
\end{aligned}
$$


Using these estimates in (2.10), we get

$$
\begin{aligned}
\|y *(t)-y(t)\| \leq & \left\|Y(t) D^{-1} \delta \alpha\right\|+\int_{a}^{t}\left\|Y(t) D^{-1} \delta M Y(a) Y^{-1}(s) P^{-1}(s) f(s)\right\| d s \\
& +\int_{t}^{b}\left\|Y(t) D^{-1} \delta N Y(b) Y^{-1}(s) P^{-1}(s) f(s)\right\| d s \\
& +\int_{t}^{b}\left\|Y(t) D^{-1} \delta R Y(c) Y^{-1}(s) P^{-1}(s) f(s)\right\| d s \\
& +\int_{t}^{b}\left\|Y(t) D^{-1} \delta S Y(d) Y^{-1}(s) P^{-1}(s) f(s)\right\| d s \\
& +\int_{b}^{c}\left\|Y(t) D^{-1} \delta R Y(c) Y^{-1}(s) P^{-1}(s) f(s)\right\| d s \\
& +\int_{b}^{c}\left\|Y(t) D^{-1} \delta S Y(d) Y^{-1}(s) P^{-1}(s) f(s)\right\| d s \\
+ & \int_{c}^{d}\left\|Y(t) D^{-1} \delta S Y(d) Y^{-1}(s) P^{-1}(s) f(s)\right\| d s \\
\leq & \left\|Y(t) D^{-1} \delta \alpha\right\|+\left\{\| Y(t) D^{-1}[\delta M Y(a)+\delta N Y(b)+2 \delta R Y(c)\right. \\
& +3 \delta S Y(d)] \|\} \int_{a}^{d}\left\|Y^{-1}(s) P^{-1}(s) f(s)\right\| d s \\
\leq & \delta \beta+\delta \beta K[\|Y(a)\|+\|Y(b)\|+2\|Y(c)\|+3\|Y(d)\|] \\
= & \delta \beta[1+
\end{aligned}
$$

The reverse in equality follows by noting the fact that

$$
\left\|y^{*}(t)-y(t)\right\| \geq\left\|Y(t) D^{-1} \alpha\right\|-\int_{a}^{d}\left\|G_{1}(t, s)-G(t, s)\right\|\|f(s)\| d s .
$$

One may choose $\beta$ such that

$$
\beta \leq \max _{t \in[a, d]}\|Y(t)\|\left\|D^{-1}\right\|
$$

to obtain a more reliable quantity for $\beta$.

The estimates in the above theorem depends on the well known quantities and the values of the fundamental matrix at the boundary points.

\section{Acknowledgement}

The author is greatful to Dr. K. N. Murty for his valuable suggestions in preparing the manuscript. 


\section{References}

[1] R. H. Cole, The Theory of Ordinary Differential Equations, Appelton-Century Crofts, 1968.

[2] R. M. M. Matheja, "The Conditioning of Linear Two Point Boundary Value Problems," SIAM J. Numerical Analysis, 19(1982), 963-978.

[3] K. N. Murty, and S. Sivasundaram, "Existence and Uniqueness and Conditioning of Three Point Boundary Value Problems Associated with a System of First Order Non-linear Differential Equations," Journal of Math. and Phy. Sci., 26(3)(1992), 267-281.

[4] M. S. N. Murty, "Non-Linear Three Point Boundary Value Problems Associated with System of First Order Matrix Differential Equations," Bull. Inst. of Mathematics, Academia Sinica, 15(2) (1987), 243-249.

Department of Applied Mathematics Nagarjuna University Postgraduate Centre Nuzvid 521 201, Andhra Pradesh India. 